\title{
FIDELITY AND THE SPEED OF THE TREADMILL: THE COMBINED IMPACT OF POPULATION SIZE, TRANSMISSION FIDELITY, AND SELECTION ON THE ACCUMULATION OF CULTURAL COMPLEXITY
}

\author{
Claes Andersson and Petter Törnberg
}

\begin{abstract}
Human culture signifies the emergence of an entirely new domain of existence: an event in natural history that is paralleled only by the Cambrian Explosion in terms of creativity and scope. The question of how human culture-as opposed to its animal counterparts - came to become open-endedly creative and cumulative is therefore one of wide and general scientific importance. Several causal factors have been proposed to date to explain this unique quality, including population size, transmission fidelity, pedagogy, and creativity. Inquiries, however, tend to focus exclusively on one factor at a time, leaving us blind to important issues regarding their relative roles and combined action. We here combine two models, one focusing on population size and the other on imitation fidelity, as constraints and enablers of evolutionary cumulativity. We explore how these factors interact to promote and inhibit evolutionary cumulativity and how the synthetic model compares to the original models individually and to empirical and experimental data. We report several findings that do not emerge in the models that we combine individually. For example, group size is found to be important for small but not for larger groups, an observation that moreover substantially improves agreement with data.
\end{abstract}

La cultura humana significa la aparición de un dominio de la existencia completamente nuevo: un acontecimiento en la historia natural sólo equivalente a la explosión cámbrica en términos de creatividad y alcance. La cuestión de cómo la cultura humana - a diferencia de sus equivalencias entre los animales - se convirtió en creativa y acumulativa se convierte por lo tanto en un tema de importancia científica amplia y general. Hasta la fecha se han propuesto varios factores causales para explicar esta cualidad única, incluyendo el tamaño de la población, la fidelidad de la transmisión, la pedagogía y la creatividad. Las investigaciones, sin embargo, tienden a centrarse exclusivamente en uno de estos factores a la vez, lo que nos deja ciegos a cuestiones importantes con respecto a sus roles relativos y a su interacción. En esta investigación combinamos dos de estos modelos, uno que se centra en el tamaño de la población y el otro en la fidelidad de imitación como restricciones y facilitadores de la acumulación evolutiva. Exploramos también cómo estos factores interactúan para promover e inhibir dicha acumulación evolutiva, cómo el modelo sintético se compara con los modelos originales de forma individual y con datos empíricos y experimentales. Presentamos varios hallazgos que no surgen en los modelos que combinamos de forma individual. Por ejemplo, se encuentra que el tamaño del grupo es importante para grupos pequeños pero no para los grupos más grandes; observación que, además, mejora sustancialmente la concordancia con los datos.

$\mathrm{T}$ The development of increasingly complex organization in human evolution is unparalleled in natural history and signifies the emergence of an entirely new domain of existence: the rise of human culture out of the domain of animal social behavior. What drove, channeled, and constrained this development has long been a subject of considerable debate, with proposed drivers and constraints including: fidelity (Andersson 2011, 2013; Claidière and Sperber 2010; Enquist et al. 2010; Tennie et al. 2009; Tomasello
1991), pedagogy (Csibra and Gergely 2009, 2011; Sterelny 2011; Tostevin 2007, 2012), time stress and environmental risk (Buchanan et al. 2015; Read 2008a; Torrence 1983, 1989), generative logic (Leaf and Read 2012; Read 2002, 2003), intensity and broadness of resource utilization (Flannery 1976; Keeley 1988), and social effects of population size (Henrich 2004; Shennan 2001).

Over the past decade, a class of cultural evolutionary models belonging to the last category has become increasingly influential (see Anders-

Claes Andersson and Petter Törnberg — Division for Physical Resource Theory, Department of Energy and Environment, Chalmers University of Technology, 41296 Göteborg, Sweden (claeand@chalmers.se, corresponding author)

American Antiquity 81(3), 2016, pp. 576-590

Copyright $(\subset) 2016$ by the Society for American Archaeology

DOI: $10.7183 / 0002-7316.81 .3 .576$ 
son and Read 2016). The most seminal among these models, put forward by Henrich (2004), is based on the argument that since the imitation of cultural skills is imperfect, a constant loss of information through imitation errors must be compensated for by the creativity of individuals in order to maintain the long-term persistence of cultural systems. Kline and Boyd (2010:2559) describe this process as a "treadmill of cultural loss."

The central prediction of this "Treadmill Model" is that large populations are needed to maintain complex cultural systems. The larger the population, Henrich (2004) argues, the more likely the presence of highly creative individuals capable of augmenting and elaborating learned skills even beyond what is needed to compensate for information loss. A larger population can, therefore, "run faster" to counteract cultural losses and accumulate skillfulness in more complex cultural traits.

Since 2004, additional theoretical (Baldini 2013; Kobayashi and Aoki 2012; Mesoudi 2011; Nakahashi 2014; Powell et al. 2009, 2010), empirical (Kline and Boyd 2010) and experimental (Derex et al. 2013; Kempe and Mesoudi 2014; Muthukrishna et al. 2014) work leads many to accept the Treadmill Model as established fact. For example, Richerson (2013:351) states with confidence that "group size determines cultural complexity" (see also Bell 2014, 2015; Chaisson 2014; Moffett 2013; Richerson et al. 2015).

But the Treadmill Model remains, at the same time, strongly contested, both theoretically and empirically, with regard to both its premises and its predictions (Collard et al. 2005; Collard, Buchanan, and O'Brien 2013; Collard, Buchanan, O'Brien, and Scholnik 2013; O'Brien and Bentley 2011; Querbes et al. 2014; Read 2006, 2008b, 2009, 2012a; Vaesen, 2012; see Andersson and Read [2016] for a review). For example, the Inuit (Read 2012b) maintained some of the most complex hunter-gatherer implements known at very low population counts - far lower than what Henrich (2004) argued caused the Tasmanians to lose the capacity to maintain much simpler bone technology and clothing.

Seen as the main driver and constraint of cultural complexity, the situation looks bleak for the Treadmill Effect. But what if we view it as one among several interacting factors?
To move in such a direction, we here build a Synthetic Model that combines key features of the Treadmill Model (Henrich 2004), which focuses on population size, with key features of the Glass Ceiling Model, which focuses on fidelity in the imitation of cultural knowledge across generations as a constraint on the accumulation of cultural complexity (Andersson 2011, 2013).

These models constitute two different perspectives on the same set of problems, and they share critical elements that allow their central features to be combined into a synthetic model. Both models investigate the conditions necessary for cultural evolution to be cumulative and for the emergence of complex cultural systems. Both give central importance to imitation fidelity. Both rely on population-based models that focus on endogenous social-level constraints on adaptive cultural evolution. And, finally, we could characterize both models as "treadmill models" since both investigate the interplay between, on the one hand, positive forces for the accumulation of novelty into complex cultural organization and, on the other hand, the negative force of losses in cultural transmission that undermine such organization. The result is a new, agent-based Synthetic Model that has three interacting variables: imitation fidelity $(q)$, population size $(N)$, and strength of selection for high-quality role models to imitate $(s)$.

We investigate the effect and interactions among these parameters in relation to data and empirically based arguments, and in relation to the combined models individually. We conclude that imitation fidelity, population size, and selection strength interact strongly and that many of their evolutionary effects become understandable only as they are studied in conjunction. We argue that the Synthetic Model provides a fuller understanding of treadmill effects more generally in cultural evolution.

\section{Synthesis}

Beginning with the Treadmill Model, we will outline the motivations, workings, and main results of each model, focusing on the central logic of each. We then move on to explain how these central logics are combined in a Synthetic Model. Formal elaborations are presented in Supplemental Materials, Appendices A-D. 


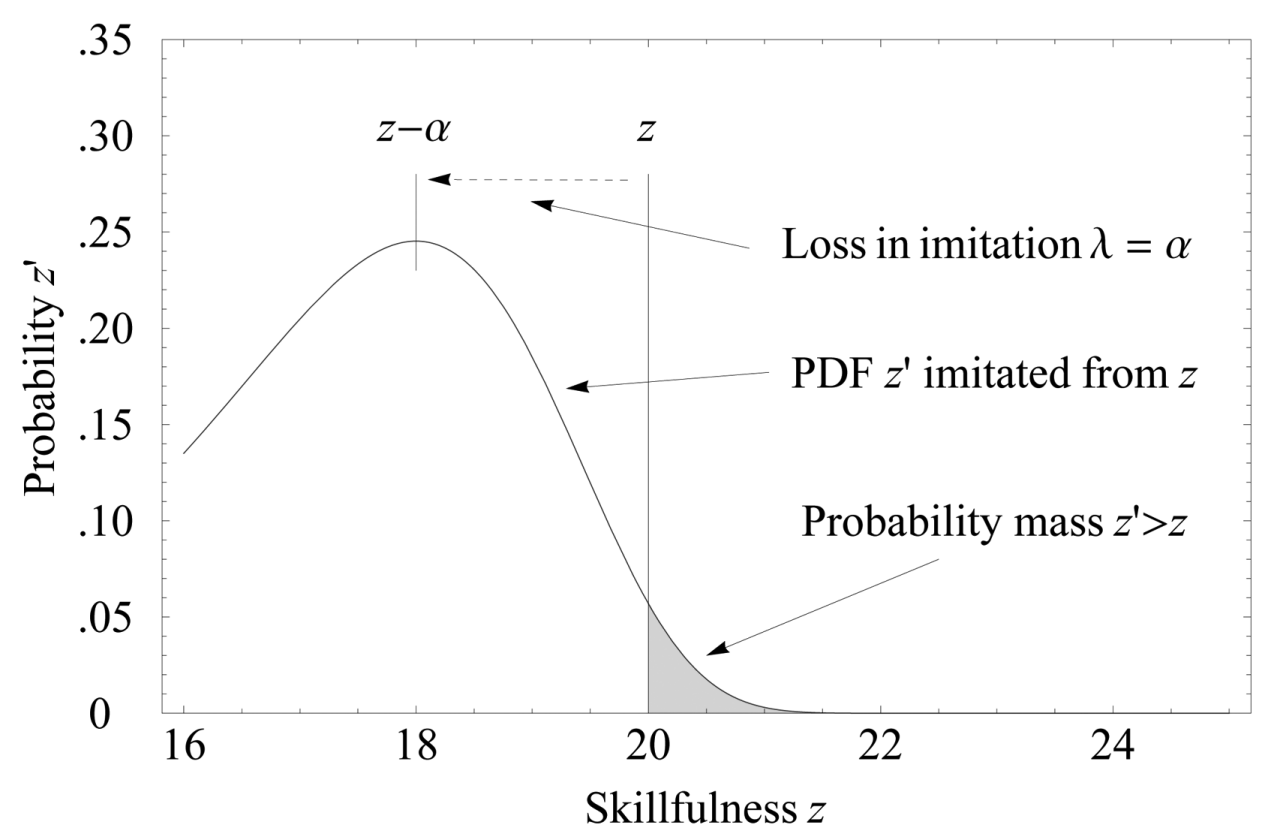

Figure 1. The probability of drawing new skillfulness values $z^{\prime}$ (vertical axis) for a naïve learner imitating a skill with complexity $\alpha=2$ from a role model with skillfulness $z=20$ (horizontal axis); see Equation 1. Stochastic variability in creativity $e$ yields a distribution of probabilities for drawing different values of $z^{\prime}=z-\alpha+e$; average creativity $(z=20)$ is the maximum-likelihood outcome. We here use the Probability Density Function of a Gumbel distribution, used by Henrich (2004) and in much of the downstream literature. The shaded area indicates the probability mass for the learner outperforming the role model (i.e., drawing $z^{\prime}>z$ ).

\section{The Treadmill Model}

The core of the Treadmill Model (Henrich 2004) is a formal mathematical model that depicts a population of "interacting social learners" undergoing what can be described as an imitation event: a new generation of naïve individuals imitate and apply their own creative capabilities to a skill possessed by a role model that represents the most highly skilled individual among already encultured members of the group.

The basic logic is that imitation combines two opposing forces that metaphorically represent (a) the backward motion of the treadmill and (b) the running that potentially keeps us from backsliding. On the one hand, high complexity of an imitated skill increases losses of skillfulness through imperfect imitation; this is because high skill complexity implies more things to learn and therefore a greater potential for mistakes and errors. On the other hand, creativity, for example hominin capabilities for invention and problem-solving, is a source of skillfulness that counteracts the source of losses related to skill complexity.
To understand what happens, let us begin from the perspective of a single naïve imitator learning from a role model. Formally we may express this as

$$
z^{\prime}=z-\alpha+e
$$

where $z$ ' denotes the skillfulness of the imitator after the imitation, $z$ the skillfulness of the role model, $\alpha$ the complexity of the imitated skill, ${ }^{1}$ and $e$ is a stochastic term that represents a varying level of creativity in individuals across the population of naïve learners. ${ }^{2}$

Since creativity is taken to affect imitation performance, heterogeneity in creativity introduces heterogeneity in skillfulness across the population, and this is what drives the Treadmill Model. The imitation event of the Treadmill Model is illustrated in Figure 1.

The question posed to the Treadmill Model is: "does an imitation event leave the population more or less skillful?" The model ultimately answers this question in the form of the rate of change per 
generation (for example, an imitation event) in average skillfulness in the population, $\Delta \bar{z}$ (Supplemental Materials, Appendix A). A positive rate of change $(\Delta \bar{z}>0)$ corresponds to cumulative adaptive evolution, while a negative rate $(\Delta \bar{z}<0)$ corresponds to maladaptive loss (see Henrich 2004). Essentially, in the maladaptive loss regime, the imitated skill is assumed to be dropped from the repertoire at the point where it becomes useless.

What decides whether a skill of complexity $\alpha$ in a population of size $N$ is viable or not becomes: will any learner, on average, at least be able to match the skillfulness of the most highly skilled role model (i.e., fall into the shaded area in Figure 1)? Figure 1 and Equation 1 tell us that the likelihood that this will be the case can be increased in two ways: (1) a lower skill complexity $\alpha$ reduces the load of imitation errors and moves the location of the imitation outcome distribution to the right, increasing the probability mass represented by the shaded area, or (2) a larger population $N$ increases the number of draws of new skillfulness values $z$, per imitation event. This increases the likelihood of producing at least one sufficiently highly skilled new role model.

The main conclusion of the Treadmill Model is that the higher the complexity of the propagated skill, the larger the population needs to be to stably maintain the skill over time. From this standpoint, we may pose the questions: how large must $N$ be to support a skill of complexity $\alpha$, or, alternatively, how complex can $\alpha$ skills be in a population of size $N ?^{3}$

Notably, the Treadmill Model applies regardless of how adaptive the skill is: if there is no sufficiently skillful role model to imitate in the population, no selection pressure in the world can compensate. ${ }^{4}$ Losses from a skill repertoire that happen in this way happen despite their adaptivity, and they are therefore described by Henrich (2004) as maladaptive losses. Henrich's (2004) main interest is whether population loss can lead to the loss of adaptive culturally transmitted skills, and he argues that the Holocene prehistory of the Tasmanian aborigines provides empirical support for such an event (see Read 2006, 2008a; Taylor 2010 for counter arguments), and the debate has continued, involving new empirical cases, model variants, and interpretations (Andersson and Read 2016).

\section{The Glass Ceiling Model}

The Glass Ceiling Model (Andersson 2011,2013) is an adaptation of the Quasispecies Model (Domingo et al. 2012; Eigen 1971; Eigen and Schuster 1977; Nilsson and Snoad 2000) to cultural evolution. Originally developed to help understand the origins of life itself, the Quasispecies Model establishes a fundamental connection between genetic replication fidelity and the amount of information that can be supported (the bandwidth, if you will) by an evolutionary population. According to the Quasispecies Model, high replication fidelity is a precondition for the emergence of long genetic sequences and thereby complex phenotypic organization. High replication fidelity in higher life forms (e.g., Drake et al. 1998) is ensured by sophisticated mechanisms of error correction, and before the establishment of such mechanisms, trait complexity was therefore strongly constrained.

The Glass Ceiling Model readapts the Quasispecies Model from questions about the origin of life to questions about the origins of culture. Has the evolution of advanced cognitive and cultural mechanisms for improving cultural transmission fidelity been as important in cultural evolution as it was in early biological and pre-biological evolution?

Although more tricky to define and measure, one can nevertheless identify innovations (cultural and biological) that would positively affect fidelity in the sense of increasing the likelihood of knowledge making it intact across generations. Examples include the capability and propensity for imitation (Gergely and Csibra 2006; Horner and Whiten 2005; Shea 2009; Whiten et al. 2009), pedagogy (Csibra and Gergely 2009, 2011; Gergely and Csibra 2006; Tehrani and Riede 2008) and cultural institutions (Andersson 2011; Henrich 2010; Sterelny 2011). But the range of cultural features that could affect cultural transmission fidelity is quite wide and includes, for instance, implicit or explicit external information storage in increasingly culturally modified environments (see Andersson 2011).

Andersson (2011) argues that since culture also relies on transmission, and since the cultural and cognitive mechanisms that counteract transmission errors and mistakes must have emerged over time 
(just as biological mechanisms maintaining high genetic transmission fidelity had to emerge as adaptations), fidelity must be expected to have played a key role also for cultural evolution. The suggestion is that human culture successively unlocked itself by increasing its bandwidth through biological and cultural innovations that increase transmission fidelity. Eventually this would lead to open-ended cultural accumulation through a bootstrapping dynamic when additional cultural complexity came to be used for innovations that further increased fidelity.

Andersson (2011) argues that seeing fidelity as an evolutionary constraint helps us explain why cultural complexity was apparently bounded across the Paleolithic, despite evidence of innovation during these periods, and despite localized evidence of precocious and more complex expressions of culture, which reveal the capacity for inventing them (Hovers and Kuhn 2006; McBrearty and Brooks 2000; McBrearty 2007). From the perspective of the Glass Ceiling Model, cognitive capabilities enabling the invention of complex innovations would be neither sufficient nor necessary to explain how cumulative cultural systems can come into being. Compare this with biological evolution where the cumulative evolution of tremendously complex organization clearly is possible, even in the complete absence of cognition. ${ }^{5}$

The central logic of the Glass Ceiling Model is that the likelihood of faithfully transmitting a skill depends strongly on how complex that skill is. Skills often constitute systems of component skills, where a high complexity $\alpha$ corresponds to skills with more components and, thereby, more internal interdependencies (Andersson 2013; Querbes et al. 2014). The idea is that component skills are learned individually but must make it across generations of learners together (due to systemic interrelations between them) in order to realize a functional high-level skill: disturbing one part potentially ruins the whole (Wimsatt 1986, 2001; Wimsatt and Griesemer 2007).

More formally, we may express the fidelity of a skill with complexity $\alpha$ as an exponential function

$$
Q=q^{\alpha}
$$

where $Q$ is the imitation fidelity of the skill as a whole, $q$ is the imitation fidelity of skill compo- nents individually (i.e., units of complexity), and $\alpha$ is the complexity of the skill as a whole.

In the evolutionary dynamics of the Glass Ceiling Model, encultured individuals $i$ in the population will possess skill copies with different complexity $\alpha_{i}$. It is complexity that is selected for (see Andersson [2013] for a discussion about what selection for complexity implies), so if the average skill complexity in the population starts out on a low level, it will initially increase. But as skill complexity increases, so does the load of imitation errors, and it does so at a rate that is determined by the imitation fidelity parameter $q$ (Equation 2).

At a certain level of average skill complexity, determined by imitation fidelity $q$, there will be an equilibrium between the adaptive force of selection pushing for higher skill complexity and the maladaptive force of imitation errors (Supplemental Materials, Appendix B). This equilibrium is the "Glass Ceiling" above, according to which too complex skills will eventually disappear even if higher complexity is both adaptive and possible to invent.

\section{The Synthetic Model}

We have introduced two models of two proposed endogenous evolutionary constraints on cultural complexity: the Treadmill Model, which is controlled by population size $N$, and the Glass Ceiling Model, which is controlled by imitation fidelity $q$. The lessons that these models convey can be summarized as formal constraints on the evolutionary dynamics (see Supplemental Materials, Appendix A, Equation 8, and Appendix B, Equation 10). Can these models be combined into a single model that retains important lessons from both but generates new insights?

Using the Treadmill Model as an envelope, we replace the sub-model $\lambda$ (Figure 1) of the displacement between role model quality and the maximum likelihood of imitation outcomes

$\begin{array}{cc} & \lambda=\alpha \\ \text { with } & \lambda=q^{-\alpha}-1\end{array}$

(Supplemental Materials, Appendix C). The Synthetic Model imitation event can be visualized as Figure 2. Note that, as in the Glass Ceiling Model, but unlike in the Treadmill Model, we consider 


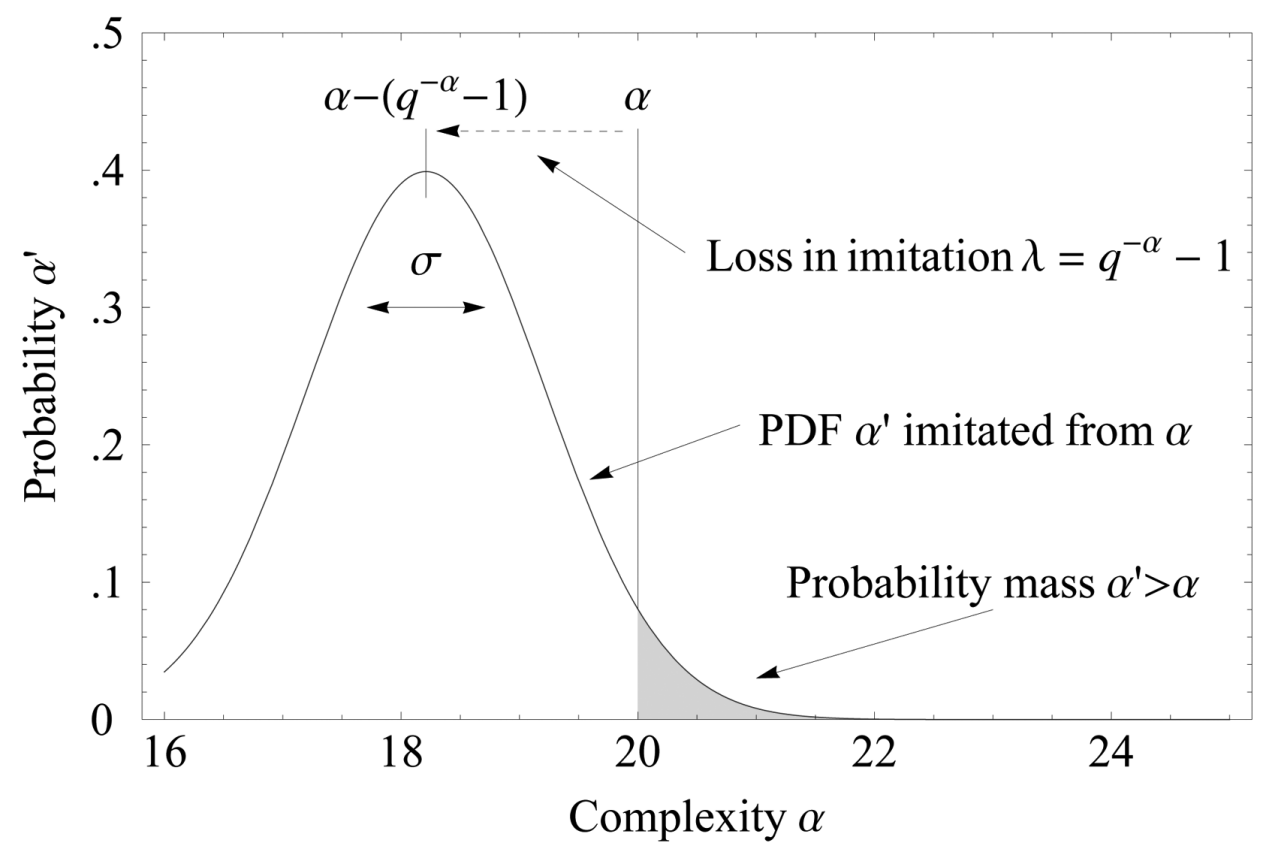

Figure 2. We illustrate the draw of a new complexity value $\alpha$ ' for a naïve learner $i$, with imitation fidelity $q_{i}$, imitating a role model with skill complexity $\alpha$. The Probability Density Function of a Normal distribution, with mean $\mu=\alpha-\left(q^{-\alpha}-\right.$ 1) and standard deviation $\sigma=1$ denotes the probability that $\alpha$ ' takes on different values. The shaded area indicates the size of the probability mass for the learner outperforming the role model (i.e., drawing $\alpha^{\prime}>\alpha$ ).

the evolution of skill complexity $\alpha$ directly $(\lambda$ changes units from $z$ to $\alpha$ between Equation 3 and Equation 4). In other words, we assume selection for increasing skill complexity (see Andersson 2013:90-91).

Variability in the Treadmill Model entered through the logic that imitators have a variable capability for counteracting imitation errors with their own creativity. Here the logic is similar, but, following the Glass Ceiling Model, it is expressed as variability in imitation fidelity $q$ instead: ${ }^{6}$ we use a stochastic fidelity $q_{i}$ that is distributed over the population. Lacking suitable empirical data, we derive a sub-model for how $q_{i}$ could be distributed by starting from the distribution of general intelligence in the population (which is typically taken to have a normal distribution), and a model parameter $q$ that is referred to as the "fidelity norm" (Supplemental Materials, Appendix C). This sub-model initiates (upon "birth") all naïve learners in the population to have their own imitation fidelity value $q_{i}$.

As in both models that go into the synthesis, selection of role models is skill biased-meaning that naïve learners favor role models based on their performance. But in contrast to Henrich (2004), who assumes that the strictly best individual acts as a role model for the whole new generation of learners, we here select role models in accordance with the Glass Ceiling Model randomly, and employing a parameter $s$ by which we may set the strength of the bias for selecting high-quality role models (i.e., the skill bias). The skill bias parameter ranges in the unit interval, $s$ $\in[0,1)$ where $s=0$ corresponds to no selection (pure drift) and $s \rightarrow 1$ corresponds to the case of the best role model, is always being selected as in the Treadmill Model (Supplemental Materials, Appendix C).

Updates are performed by letting a new generation of naïve learners select role models to imitate among already encultured individuals. Imitation generates a complexity $\alpha_{i}$ in the imitator from a normal distribution with mean

$$
\mu=\alpha_{r}-\lambda
$$

and standard deviation $\mathrm{s}=1$, where $\alpha_{r}$ is the skill complexity of the selected role model, and where 


$$
\lambda=q_{i}^{-\alpha}{ }_{r}-1
$$

is the average imitation error (Figure 2).

This Synthetic Model allows us to investigate the interaction between three important hypothetical factors affecting the cultural accumulation of skills: population size $(N)$, skill bias strength $(s)$, and imitation fidelity $(q)$.

Figure 3 illustrates the basic ways in which changes in these three parameters affect the evolutionary dynamics of skill complexity $\alpha$ are in the population (see also Supplemental Materials, Movies 1-4). If we increase population size $N$ (Figure 3b), individual skill complexity values $\alpha_{i}$ will scatter more widely since we make more "draws." If we selectively choose highly skilled role models, this will, in turn, lead to a higher average skill complexity $\alpha$ of selected role models. However, higher values decrease rapidly in frequency in the tail of a normal distribution, so in order to keep increasing, average $\alpha$ among role models $N$ needs to increase more and more.

As we increase skill bias $s$ (Figure 3c), we become more strict in selecting role models from the right side of the distribution. This has the effect of increasing average skill complexity among the selected role models, and thereby in the new generation. However, increasing $s$ will work only as long as there actually is any variation upon which stricter selection standards can operate: if we already select the best, stricter selection will not take us any farther.

If we increase the fidelity norm $q$ (Figure 3d) we reduce the average error $\lambda$ (Equations 5, 6 and Figure 2), which increases exponentially with skill complexity $\alpha$, and so we also increase average skill complexity in the new generation in a third way. This strategy allows us to increase skill complexity indefinitely, since at $q=1$ the error will be zero. However, it must realistically be assumed that reducing error becomes increasingly hard, so approaching $q=1$ may demand considerable adaptations and costs.

\section{Results}

We will now use simulation to investigate the interplay between population size $N$, the imitation fidelity norm $q$, and the strictness of skill bias in role model selection $s$. The simulated populations are initiated with parameter values that remain fixed while a sufficient number of updates are performed to bring the average skill complexity $\alpha$ in the population to equilibrium.

Both the sensitivity of equilibrium average $\alpha$ to skill bias strength $s$, and the impact of $s$ on how $\alpha$ responds to changes in $N$, clearly emerge in Figure 4. Only when those rare geniuses really have an edge will increasing population size $N$ have anything like a sustained effect. The qualitative similarity between the simulated behavior of the Synthetic Model and the predictions of the original Treadmill Model also increases with skill bias strength $s$, which is unsurprising given that the Treadmill Model assumes perfect skill bias $s$ $=1$. But the synthetic model, notably, does not collapse into the Treadmill Model as $\mathrm{s} \rightarrow 1$. Even at $s=.99$, the response to increasing $N$ abates rapidly, and the increase of the logarithmic Treadmill curve is more sustained than the simulation results of the Synthetic Model.

The response of the Synthetic Model to changes in population size $N$ (Figure 4) can be understood as an interaction between the Treadmill Model, which predicts a logarithmic dependency between $\alpha$ and population size $N$ (Supplemental Materials, Appendix A, Equation 8); the Glass Ceiling Model, which strictly bounds $\alpha$ subject to transmission fidelity (Supplemental Materials, Appendix B, Equation 10) independently of $N$, and the skill bias strength $s$, which affects the average skill complexity of the role models that are imitated. What we see, at first, is that increasing $N$ causes average $\alpha$ to increase, as predicted by the Treadmill Model. But already at a comparably low value of $N$, equilibrium average $\alpha$ becomes bounded by the Glass Ceiling effect instead, causing it to deviate from the logarithmic Treadmill relationship and level off. We will revisit the effect of skill bias strength $s$ shortly.

Next, the response to varying imitation fidelity $q$ is strong and qualitatively very similar to the response seen in the original Glass Ceiling Model (Figure 5; Andersson 2011, 2013). It is not surprising that imitation fidelity $q$ has a commanding effect: in the limit case of $q=1$ the average error is zero regardless of $\alpha$, and the logic of both models that we combine would clearly predict unlimited cultural accumulation under that (unrealistic) condition. 
$\odot$ Individuals $\alpha_{i}$ in current population

- Individuals selected as role models

Average skill complexity in population before

Average skill complexity among selected role models

,- Average skill complexity in population after imitation

$\leftarrow$ Average imitation error $\left(\lambda=q^{-\alpha}-1\right)$

$\Rightarrow$ Change in average skill as a result of imitation

\section{Light color in (b-d) indicate base case}

(a) Base case

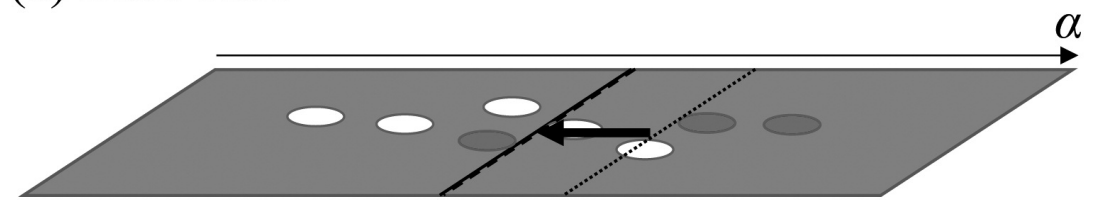

(b) Increasing $N$

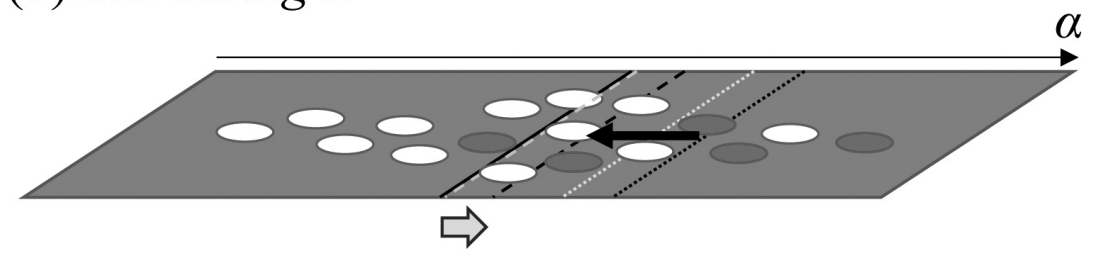

(c) Increasing $s$

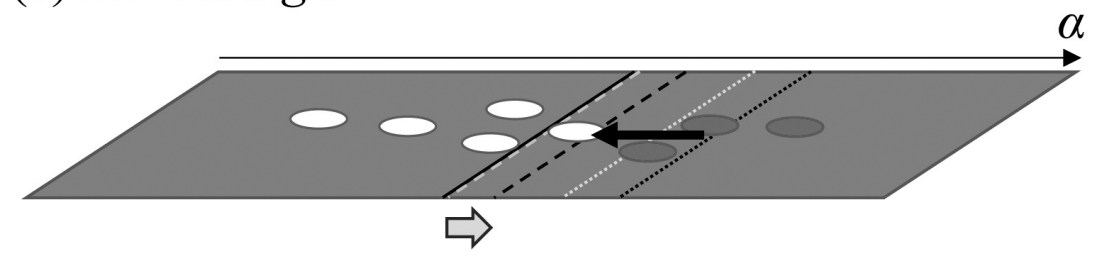

(d) Increasing $q$

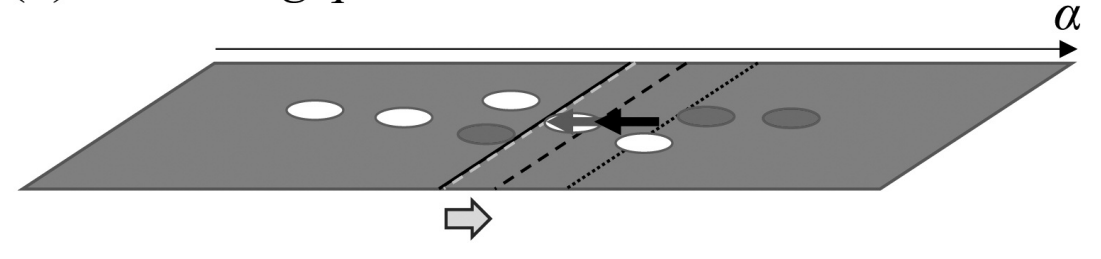

Figure 3. Individuals $\left(\alpha_{i}\right)$ are distributed across the skill complexity $\alpha$ axis, with skill biased selection favoring role models with large values of $\alpha_{i}$. In the base case (a), average $\alpha$ after imitation is the same as average $\alpha$ before imitation. In (b-c) we upset this equilibrium, driving the population to higher average skill complexity by altering, in turn, population size $N$, skill bias strength $s$ and imitation fidelity $q$. See also Movies in the Supplemental Materials. 


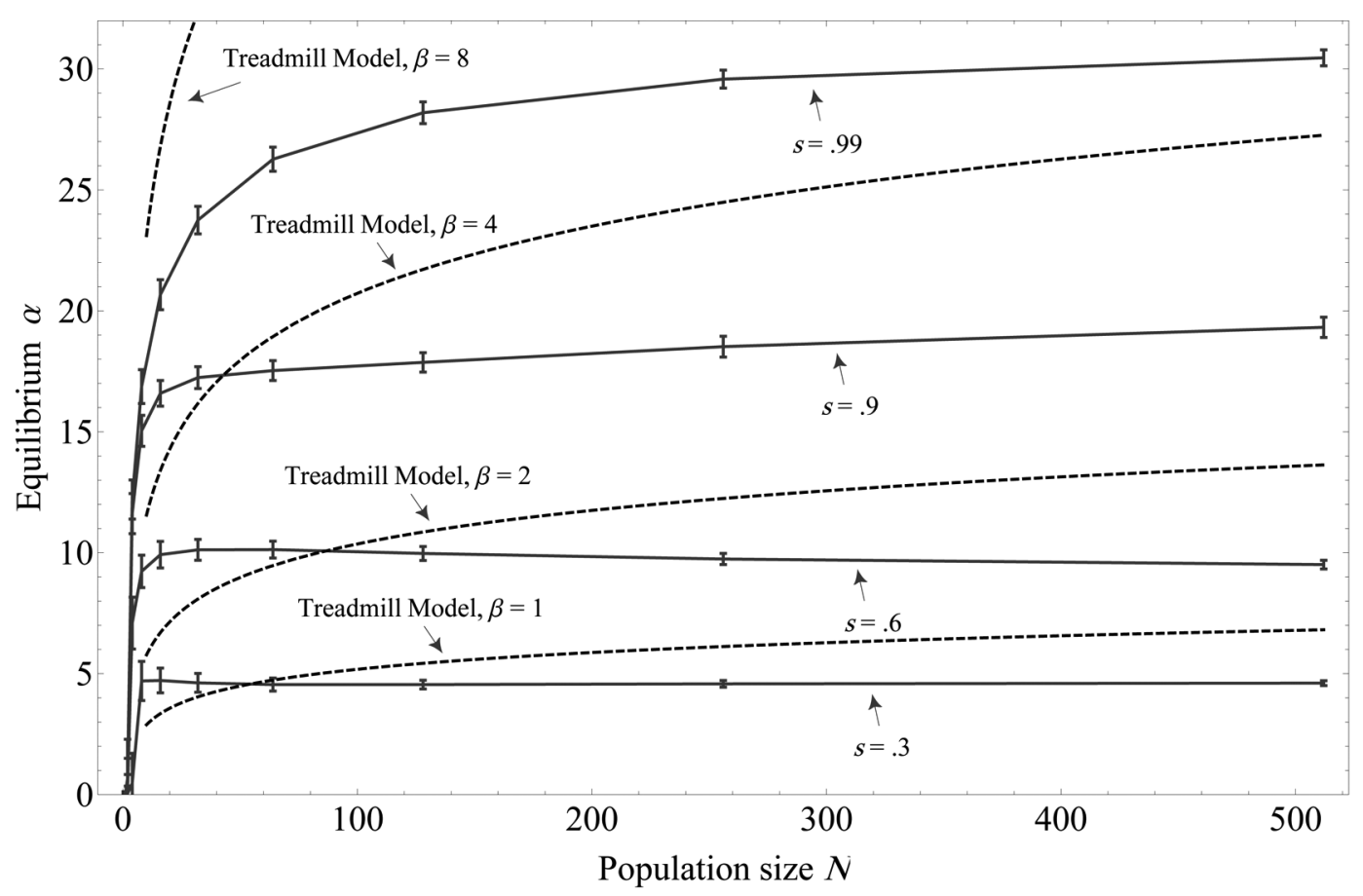

Figure 4. Average equilibrium skill complexity $\alpha$ plotted as a function of interacting learning population size $N$, and for different values of skill bias strength $s$. For comparison, we also see predictions by the original Treadmill Model (see Equation 8 in Appendix A in Supplemental Materials), using its $\beta$ parameter (which we otherwise suppress) to examine different scales of $\alpha$ (its units are arbitrary, i.e., do not correspond to empirical measures). Parameters used: $q=.95$, simulations run over 10,000 updates, averages made over batches of 50 such runs, error bars correspond to one standard deviation. Population sizes used were $N=2,4,8,16,32,64,128,256,512$.

A strong skill bias generally increases equilibrium average skill complexity $\alpha$ (Figures 4,5 ). Figure 6 shows that skill bias has a sustained impact at higher values of imitation fidelity $q$, but that for lower imitation fidelity, the response grows notably weaker. Using a population size of $N=5$ rather than $N=25$, we further see that this pattern also depends on population size $N$ : the smaller the population, the weaker the response (Figure $6 \mathrm{~b})$. This is readily understandable in terms of evolutionary dynamics: the smaller the population, the less likely is the appearance of highly gifted individuals, and skill bias $s$ can push equilibrium skill complexity $\alpha$ upward only to the extent that a chain of such skilled individuals connects the generations through time. Highly skilled individuals who appear only sporadically will frequently need to imitate less skilled role models, and so their impact will not be lasting.

Turning now to the parameter space where equilibrium average $\alpha$ does respond strongly to
$N$, (Figure 7; compare Figure 5) we see that increasing group size beyond $N=25$, which would be the relevant range for hunter-gatherer residential groups, does not produce a strong effect. However, lowering population size to below $N=10$ does bring a substantial dip in equilibrium average $\alpha$. So, even if the Treadmill effect is suppressed by the Glass Ceiling Effect for large populations, it could represent an important constraint on the size of residential units, and represent one reason why increasing sizes of residential units may have been favored by selection. ${ }^{7}$

We have also superimposed scaled data from Derex et al. (2013:Figure 3), describing the performance of experimental groups of different sizes. It is notable that the qualitative behavior of the experimental and simulated curves are similar: performance increases for small groups, then levels off sharply around a group size of $N \sim 10$. Their data appear to be consistent with the Synthetic Model for a low degree of skill bias, while 


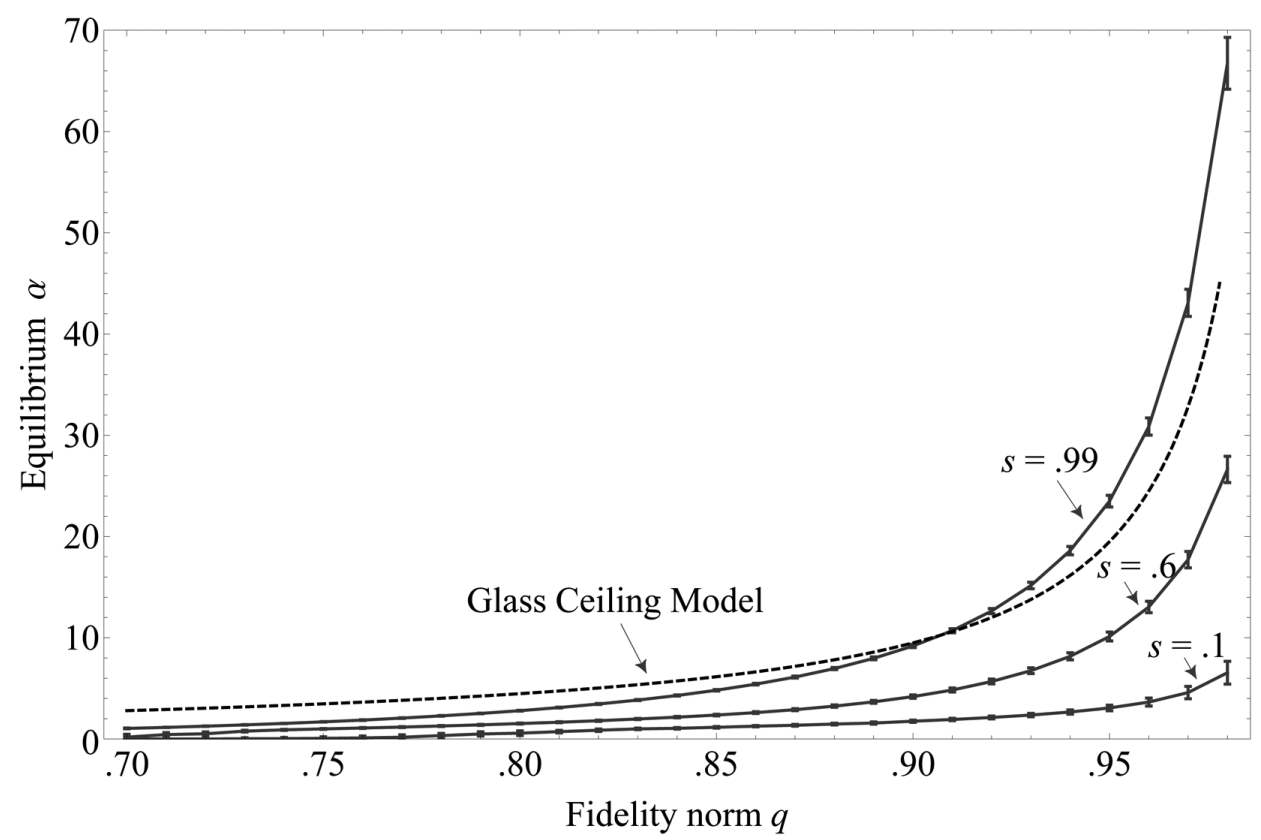

Figure 5. Skill complexity $\alpha$ plotted as a function of fidelity norm $q$, and for different values of skill bias strength $s$. For comparison, we also see the prediction of the original Glass Ceiling Model (Equation 10 in Appendix B in Supplemental Materials). Parameters used: $N=25$ simulations run over 10,000 updates, averages made over batches of 50 such runs, error bars correspond to one standard deviation.

the fact that experimental performance actually deteriorates between $N=8$ and $N=16$ clearly points away from the sustained logarithmic increase predicted by the Treadmill Model.

One of the main issues in the literature so far has been the historical impact of changes in population size, that is, changes that happen as cultural evolution unfolds over time. An exploration of how simulated histories respond to parameter change events is provided in Supplemental Materials, Appendix D.

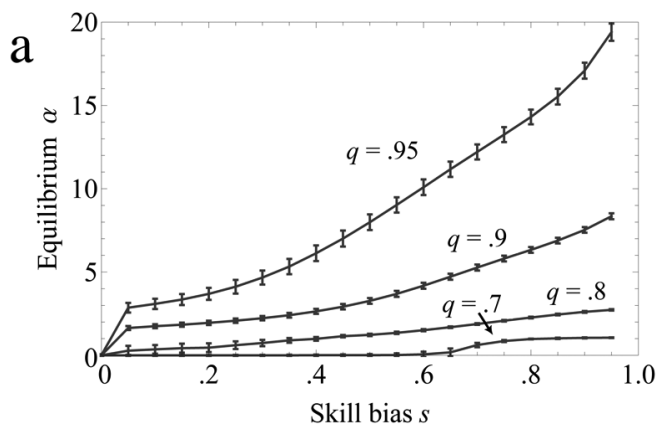

\section{The Empirical and Experimental Picture}

To discuss the Synthetic Model's contributions, let us first reflect on what theoretical account the empirical and experimental picture can support at present. We focus on complexity and population size, since this is the correlation that has so far been extensively investigated in the literature (Andersson and Read 2016).

It appears well established experimentally that group skill performance does tend to increase with

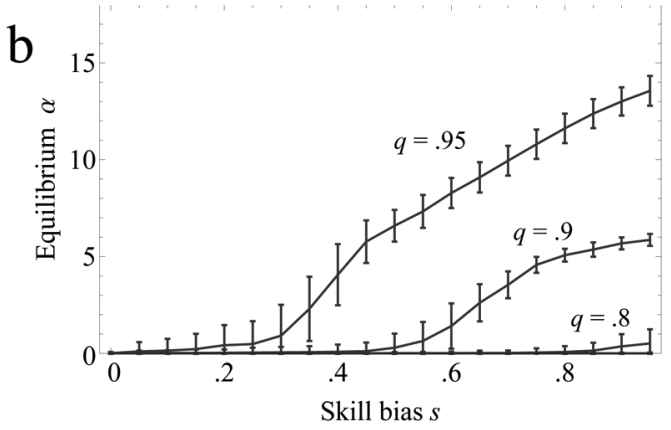

Figure 6. Skill complexity $\alpha$ plotted as a function of skill bias strength $s$, for different values of imitation fidelity norm $q$, and for two population size scenarios: (a) $N=25$ and (b) $N=5$. Simulations run over 10,000 updates, averages made over batches of 50 such runs, error bars correspond to one standard deviation. 


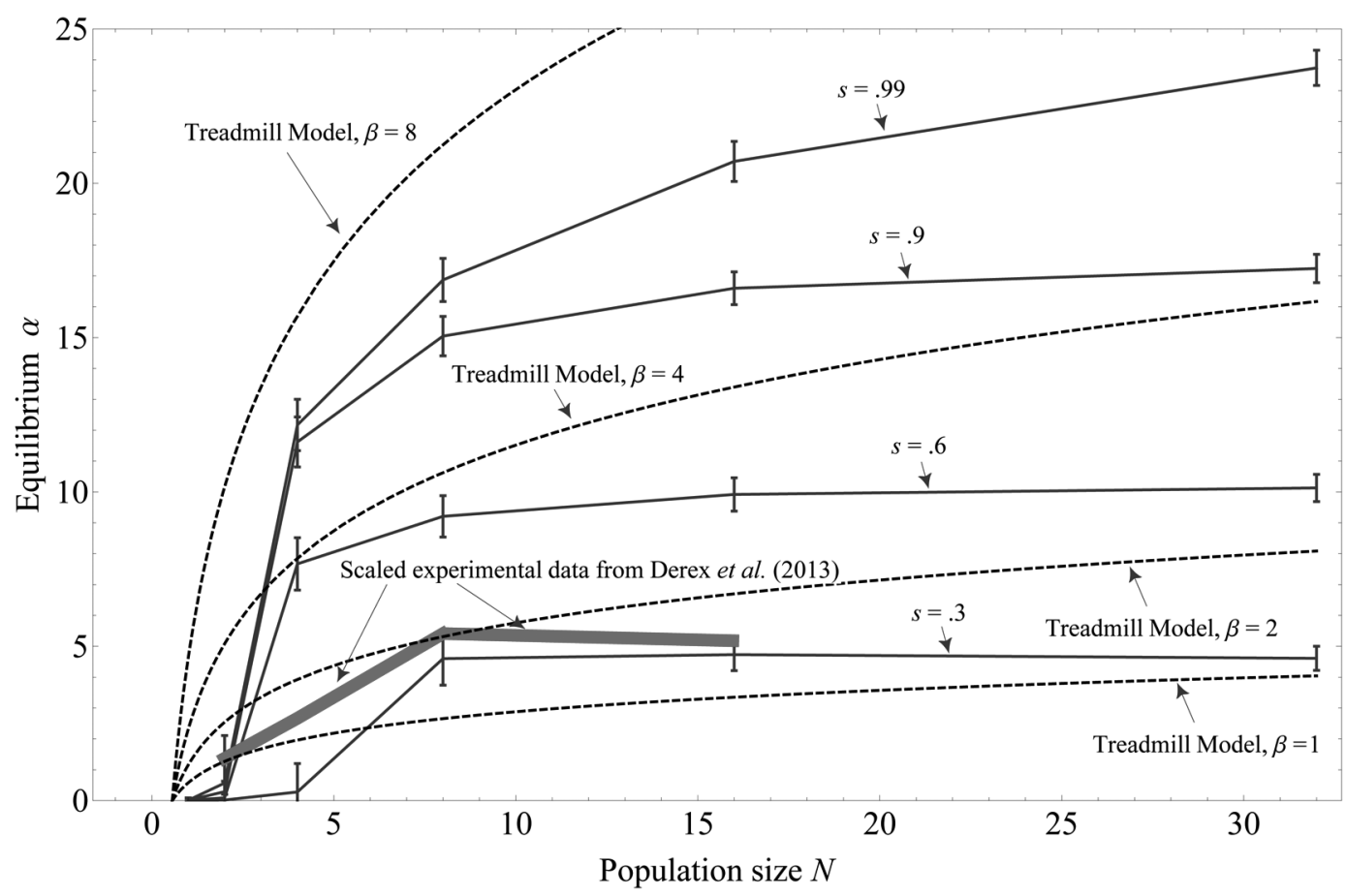

Figure 7. Zoomed-in portion of Figure 4, showing changes in equilibrium average $\alpha$ for smaller group sizes. Scaled data is shown from Derex et al. (2013), describing performance of experimental groups of different sizes. Parameters used: $q$ $=.95$, simulations run over 10,000 updates, averages made over batches of 50 such runs, error bars correspond to one standard deviation. Population sizes used were $N=2,4,8,16,32$.

group size for small groups, on the order of one to ten individuals (see Derex et al. 2013; Kempe and Mesoudi 2014; Muthukrishna et al. 2014). Although it remains somewhat inconclusive whether it is the specific mechanisms proposed by the Treadmill Model that are responsible for the observed behavior, data on small groups is at least consistent with predictions of the Treadmill Model (e.g., Andersson and Read 2014).

However, for meta-populations the Treadmill Model runs into trouble (Buchanan et al. 2015; Collard, Buchanan, and O'Brien 2013; Collard, Buchanan, O'Brien, and Scholnik 2013; Read 2008a). Several recent studies fail to demonstrate any significant correlation between population size estimations and measures of cultural complexity (based on Oswalt's [1976] techno-units). Read (2008b) shows that over 95 percent of the variability in hunter-gatherer food-getting implements relates to mobility and risk. There is also no correlation between either the census population size or the population density and complexity of tool kits for hunter-gatherer societies across major environmental and ecological zones (see Collard et al. 2005; Collard, Buchanan, O'Brien, and Scholnik 2013; Read 2006).

Demonstrating these troubles even more clearly, there exist notable empirical counterexamples. For example, the Angmaksalik of eastern Greenland, a group of about 400 Inuit (Read 2012a), made a 36-part harpoon, which counts among the most complex implement manufactured by any hunter-gatherer group. Even if we unrealistically assume that the total population of about 6,000 Inuit in all of Greenland constituted the "population of interacting social learners" (Henrich 2004:202), they still numbered considerably lower than what Henrich (2004) posits was needed for the Tasmanians to even make one-part bone point implements.

The experiment performed by Derex et al. (2013), which is the experiment that most closely corresponds to the Treadmill Model, and the only experiment so far to consider sufficiently large group sizes, also falls into this pattern. Performance here leveled off, and even deteriorated, be- 
tween populations sizes of $N=8$ and $N=16$ (see Figure 7; also see Derex et al. 2013:Figures 1-3; Derex et al. 2014:Figure 1).

The empirical and experimental picture points to population size correlating with skill complexity only for very small groups. This is also what the Synthetic Model predicts (see Figures 4 and 7), and explains by mixing models of transmission fidelity, less-than-perfect skill bias and population size.

Empirically, there are indications that residential group sizes may have increased from very small numbers during the course of hominin evolution, and this may indicate that the Treadmill Model could be more important for understanding early hominin evolution than it is for understanding variation in cultural complexity among recent hunter-gatherers. Vallverdú et al. (2010:143) and Lalueza-Fox et al. (2011:250), for example, indicate that Neanderthals may have traveled in groups of around 10 individuals (although estimations of residential group size in the deep past are far from secure, and these findings are controversial; see Vigilant and Langergraber 2011). Although further research is needed, evolution does document a trend from small and transient to larger and more stable groups in hominin evolution.

\section{Conclusions}

The Synthetic Model can be understood in outline as an agent-based rendition of the Treadmill Model where transmission error rate increases exponentially rather than linearly (see Equations 3 and 4) with increasing skill complexity, and where the strictness of skill bias in role model selection is controlled by a parameter rather than being assumed to be perfect.

We find that the accumulation of cultural complexity is constrained in different and interacting ways by population size $N$, imitation fidelity $q$, and the strictness of skill bias in role model selection $s$. We recognize the behavior of both the Treadmill Model (Henrich 2004) and the Glass Ceiling Model (Andersson 2011, 2013), but many of the results could not have arisen in models that focus on single factors. This underscores the need, in general, to explore interactions between proposed explanatory factors. Doing so here we found that (a) keeping population size $N$ stable and in- creasing imitation fidelity $q$ will increase skill complexity $\alpha$ without limits for any non-zero values of $N$ and $s$; see Figure 5,(b) keeping imitation fidelity $q$ stable and increasing population size $N$ increases skill complexity $\alpha$ for small population sizes (up to a few tens of individuals) but not for larger populations, and (c) increasing skill bias strength $s$ will increase skill complexity $\alpha$ for any population size $N$ and imitation fidelity $q$, but only up to a point; see Figure 6 . The basic mechanisms by which the model responds to changes in these parameters were explained above, in "The Synthetic Model," and in Figure 3; see also Supplemental Material, Movies.

Our synthetic model shows that population size is likely a strong constraint on cultural accumulation for small groups (up to 10-20 members), but the effect abates rapidly for larger groups (see Figures 4 and 7), which was the context of the original claims by Henrich (2004). This abatement becomes highly pronounced when we look at skill bias strictness levels that are less than perfect, as they must be assumed to have been in realistic contexts. This is also in agreement with the current empirical and experimental data.

The Synthetic Model also shows that the strictness of skill bias in role model selection determines how well a population can "make use of" large numbers. If skill bias (see Supplemental Materials, Appendix C, Equation 13) is strong, then increasing population size will have a more sustained effect on cultural accumulation (see Figure 5 and Supplemental Material, Appendix D, Figure 12). However, maintaining a strong skill bias would appear to become progressively harder the larger and more dispersed a population gets: How do you find the best role models? How do you ensure that imitators get to spend sufficient time in close interaction with them (Tostevin 2007) to learn complex skills?

Imitation fidelity can be likened to a lever that controls the speed of the Treadmill (at perfect imitation, the Treadmill would be at rest): it determines the rate at which loss-rates in imitation grow as skill complexity increases. Cultural and biological innovation with the effect of improving fidelity can be identified. Finally, imitation fidelity has a commanding effect on cultural accumulation. Cultural evolutionary open-endedness could occur once fidelity-induced additional capacity 
for cultural complexity came to be sufficiently reinvested in innovations that further increased transmission fidelity. ${ }^{8}$

Acknowledgments. Funding was provided by the EC FP7 project MD (\#284625). We wish to thank Dwight Read, with whom we have interacted strongly on the topics that this modeling work addresses, as well as the editor and two anonymous reviewers who contributed insightful and in-depth comments. Luis Borrero checked the Spanish abstract.

Supplemental Materials. Supplemental materials are linked to the online version of this paper, which is accessible via the SAA member login at www.saa.org/members-login.

Supplemental Text 1. Appendices A-D and Overview, Design Concepts, and Details (ODD) of the Model. This document also includes links to movies on YouTube.

\section{References Cited}

Andersson, Claes

2011 Palaeolithic Punctuations and Equilibria: Did Retention Rather Than Invention Limit Technological Evolution? PalaeoAnthropology 2011:243-259.

2013 Fidelity and the Emergence of Stable and Cumulative Sociotechnical Systems. PalaeoAnthropology 2013:88103.

Andersson, Claes, and Dwight W. Read

2014 Group Size and Cultural Complexity. Nature 511(7507):E1

2016 The Evolution of Cultural Complexity: Not by the Treadmill Alone. Current Anthropology 57(3):261-286.

Baldini, Ryan

2013 Revisiting the Effect of Population Size on Cumulative Cultural Evolution. Journal of Cognition and Culture 15: 320-336.

Bell, Adrian V.

2014 Cultural Evolution and the Way We Count. Proceedings of the National Academy 111:1227-1228.

2015 Linking Observed Learning Patterns to the Evolution of Cultural Complexity. Current Anthropology 56:277281.

Buchanan, Briggs, Michael J. O'Brien, and Mark Collard

2015 Drivers of Technological Richness in Prehistoric Texas: An Archaeological Test of the Population Size and Environmental Risk Hypotheses. Archaeological and Anthropological Sciences 1-10. DOI 10.1007/s12520-0150245-4.

Chaisson, Eric J.

2014 Practical Applications of Cosmology to Human Society. Natural Science 6:767-796.

Claidière, Nicolas, and Dan Sperber

2010 Imitation Explains the Propagation, Not the Stability of Animal Culture. Proceedings of the Royal Society of London B: Biological Sciences 277(1681):651-659.

Collard, Mark, Briggs Buchanan, and Michael J. O'Brien

2013 Population Size as an Explanation for Patterns in the Paleolithic Archaeological Record. Current Anthropology 54(S8):S388-S396.

Collard, Mark, Briggs Buchanan, Michael J. O'Brien, and Jonathan Scholnick

2013 Risk, Mobility or Population Size? Drivers of Tech- nological Richness Among Contact-Period Western North American Hunter-Gatherers. Philosophical Transactions of the Royal Society B: Biological Sciences 368(1630): 20120412.

Collard, Mark, Michael Kemery, and Samantha Banks

2005 Causes of Toolkit Variation among Hunter-Gatherers: A Test of Four Competing Hypotheses. Canadian Journal of Archaeology/Journal Canadien d'Archéologie 29(1):119.

Csibra, Gergely, and György Gergely

2009 Natural Pedagogy. Trends In Cognitive Sciences 13(4): 148-153.

2011 Natural Pedagogy as Evolutionary Adaptation. Philosophical Transactions of the Royal Society of London. Series B, Biological Sciences 366(1567):1149-1157.

Derex, Maxime, Marie-Pauline Beugin, Bernard Godelle, and Michel Raymond

2013 Experimental Evidence for the Influence of Group Size on Cultural Complexity. Nature 503(7476):389391.

2014 Derex et al. Reply. Nature 511(7507):E2.

Domingo, Esteban, Julie Sheldon, and Celia Perales

2012 Viral Quasispecies Evolution. Microbiology and Molecular Biology Reviews 76(2):159-216.

Drake, John W., Brian Charlesworth, Deborah Charlesworth, and James F. Crow

1998 Rates of Spontaneous Mutation. Genetics 148:16671686.

Eigen, Manfred

1971 Self-Organization of Matter and the Evolution of Biological Macromolecules. Naturwissenschaften 58(10):465523.

Eigen, Manfred, and Peter Schuster

1977 The Hypercycle. A Principle of Natural Self-Organization. Naturwissenschaften 64(11):541-565.

Enquist, Magnus, Pontus Strimling, Kimmo Eriksson, Kevin Laland, and Jonas Sjöstrand

2010 One Cultural Parent Makes No Culture. Animal Behaviour 79(6): 1353-1362.

Flannery, Kent V.

1976 The Early Mesoamerican Village. Academic Press, New York.

Gergely, György, and Gergely Csibra

2006 Sylvia's Recipe: The Role of Imitation and Pedagogy in the Transmission of Human Culture. In Roots of Human Sociality: Culture, Cognition, and Human Interaction, edited by Nicholas J. Enfield and Stephen C. Levinson, pp. 229-255. Berg Publishers, Oxford, United Kingdom.

Henrich, Joseph

2004 Demography and Cultural Evolution: How Adaptive Cultural Processes Can Produce Maladaptive Losses: The Tasmanian case. American Antiquity 69(2):197-214.

2010 The Evolution of Innovation-Enhancing Institutions. In Innovation in Cultural Systems: Contributions from Evolutionary Anthropology, edited by Michael J. O'Brien and Stephen J. Shennan, pp.99-120. MIT Press, Cambridge, Massachusetts.

Horner, Victoria, and Andrew Whiten

2005 Causal Knowledge and Imitation/Emulation Switching in Chimpanzees (Pan troglodytes) and Children (Homo sapiens). Animal Cognition 8(3):164-181.

Hovers, Erella, and Steven L. Kuhn (editors)

2006 Transitions before the Transition: Evolution and Stability in the Middle Paleolithic and Middle Stone Age. Springer Science, New York.

Keeley, Lawrence H.

1988 Hunter-Gatherer Economic Complexity and "Popu- 
lation Pressure": A Cross-Cultural Analysis. Journal of Anthropological Archaeology 7(4):373-411.

Kempe, Marius, and Alex Mesoudi

2014 An Experimental Demonstration of the Effect of Group Size on Cultural Accumulation. Evolution and Human Behavior 35(4):285-290.

Kline, Michelle A., and Robert Boyd

2010 Population Size Predicts Technological Complexity in Oceania. Proceedings of the Royal Society B: Biological Sciences 277(1693):2559-2564.

Kobayashi, Yutaka, and Kenichi Aoki

2012 Innovativeness, Population Size and Cumulative Cultural Evolution. Theoretical Population Biology 82(1):3847.

Lalueza-Fox, Carles, Antonio Rosas, Almudena Estalrrich, Elena Gigli, Paula F. Campos, Antonio García-Tabernero, Samuel García-Vargas, Federico Sánchez-Quinto, Oscar Ramírez, Sergi Civit, Markus Bastir, Rosa Huguet, David Santamaría, M. Thomas P. Gilbert, Eske Willerslev, and Marco de la Rasilla

2011 Genetic Evidence for Patrilocal Mating Behavior among Neandertal Groups. Proceedings of the National Academy of Sciences 108(1):250-253.

Leaf, Murray J., and Dwight W. Read

2012 Human Thought and Social Organization: Anthropology on a New Plane. Lexington Books, Lanham, Maryland.

McBrearty, Sally

2007 Down with the Revolution. In The Human Revolution Revisited, edited by Paul A. Mellars, Katie Boyle, Ofer Bar-Yosef, and Christopher Stringer, pp. 133-151. McDonald Institute for Archaeological Research, Cambridge, United Kingdom.

McBrearty, Sally, and Alison S. Brooks

2000 The Revolution That Wasn't: A New Interpretation of the Origin of Modern Human Behavior. Journal of Human Evolution 39:453-563.

Mesoudi, Alex

2011 Variable Cultural Acquisition Costs Constrain $\mathrm{Cu}-$ mulative Cultural Evolution. PloS one 6(3):e18239.

Moffett, Mark W.

2013 Human Identity and the Evolution of Societies. Human Nature 24(3):219-267.

Muthukrishna, Michael, Ben W. Shulman, Vlad Vasilescu, and Joseph Henrich

2013 Sociality Influences Cultural Complexity. Proceeding of the Royal Society of London B: Biological Sciences 281:20132511.

Nakahashi, Wataru

2014 The Effect of Cultural Interaction on Cumulative Cultural Evolution. Journal of Theoretical Biology 352:615.

Nilsson, Martin, and Nigel Snoad

2000 Error Thresholds for Quasispecies on Dynamic Fitness Landscapes. Physical Review Letters 84(1):191-194.

O'Brien, Michael J., and R. Alexander Bentley

2011 Stimulated Variation and Cascades: Two Processes in the Evolution of Complex Technological Systems. Journal of Archaeological Method and Theory 18:309335.

Oswalt, Wendell H.

1976 An Anthropological Analysis of Food-Getting Technologies. Wiley, New York.

Powell, Adam, Stephen Shennan, and Mark G. Thomas

2009 Late Pleistocene Demography and the Appearance of Modern Human Behavior. Science 324(5932):12981301.
2010 Demography and Variation in the Accumulation of Culturally Inherited Skills. In Innovation in Cultural Systems: Contributions from Evolutionary Anthropology, edited by Michael J. O'Brien and Stephen Shennan, pp. 137-160. MIT Press, Cambridge, Massachusetts.

Querbes, Adrien, Krist Vaesen, and Wybo Houkes

2014 Complexity and Demographic Explanations of $\mathrm{Cu}-$ mulative Culture. PloS one 9(7):e102543.

Read, Dwight W.

2002 A Multitrajectory, Competition Model of Emergent Complexity in Human Social Organization. Proceedings of the National Academy of Sciences 99(Suppl. 3):72517256.

2003 The Emergence of Order from Disorder as a Form of Self Organization. Computational and Mathematical Organization Theory 9(3):195-225.

2006 Tasmanian Knowledge and Skill: Maladaptive Imitation or Adequate Technology? American Antiquity 71:164 184.

2008a Artifact Classification: A Conceptual and Methodological Approach. Left Coast Press, Walnut Creek, California.

2008b An Interaction Model for Resource Implement Complexity Based on Risk and Number of Annual Moves. American Antiquity 73:599-625.

2009 The Misuse of a Mathematical Model: The Tasmanian Case (Reply to Henrich's Response). UCLA: Human Complex Systems.

2012a Population Size Does Not Predict Artifact Complexity: Analysis of Data from Tasmania, Arctic Hunter-Gatherers, and Oceania. UCLA: Human Complex Systems.

2012b How Culture Makes Us Human: Primate Social Evolution and the Formation of Human Societies. Left Coast Press, Walnut Creek, California.

Richerson, Peter J.

2013 Group Size Determines Cultural Complexity. Nature 503:351-352.

Richerson, Peter, Ryan Baldini, Adrian Bell, Kathryn Demps, Karl Frost, Vicken Hillis, Sarah Mathew, Nicole Narr, Lesley Newson, Emily Newton, Cody Ross, Paul Smaldino, Timothy Waring, and Matthew R. Zefferman

2015 Cultural Group Selection Plays an Essential Role in Explaining Human Cooperation: A Sketch of the Evidence. Behavioral and Brain Sciences (39):e30.

Shea, Nicholas

2009 Imitation as an Inheritance System. Philosophical Transactions of the Royal Society of London. Series B, Biological Sciences 364(1528):2429-2443.

Shennan, Stephen

2001 Demography and Cultural Innovation: A Model and Its Implications for the Emergence of Modern Human Culture. Cambridge Archeological Journal 11:5-16.

Sterelny, Kim

2011 From Hominins to Humans: How Sapiens Became Behaviourally Modern. Philosophical Transactions of the Royal Society of London. Series B, Biological Sciences 366(1566):809-822.

Taylor, Timothy

2010 The Arti?cial Ape: How Technology Changed the Course of Human Evolution. Palgrave Macmillan, New York.

Tehrani, Jamshid J., and Felix Riede

2008 Towards an Archaeology of Pedagogy: Learning, Teaching and the Generation of Material Culture Traditions. World Archaeology 40:316-331.

Tennie, Claudio, Josep Call, and Michael Tomasello 2009 Ratcheting up the Ratchet: On the Evolution of $\mathrm{Cu}-$ 
mulative Culture. Philosophical Transactions of the Royal Society of London. Series B, Biological Sciences 364(1528):2405-2415.

Tomasello, Michael

1991 Chimpanzee Culture? National Student Speech Language Hearing Association Journal 18:73-75.

Torrence, Robin

1983 Time Budgeting and Hunter-Gatherer Technology. In Hunter-Gatherer Economy in Prehistory, edited by G. Bailey, pp. 11-22. Cambridge University Press, Cambridge, Massachusetts.

1989 Re-Tooling: Towards a Behavioral Theory of Stone Tools. In Time, Energy and Stone Tools, edited by Robin Torrence, pp. 57-66. Cambridge University Press, Cambridge, Massachusetts.

Tostevin, Gilbert B

2007 Social Intimacy, Artefact Visibility and Acculturation Models of Neanderthal Modern Human Interaction. In Rethinking the Human Revolution: New Behavioural and Biological Perspectives on the Origin and Dispersal of Modern Humans, edited by Paul A. Mellars, pp. 341357. McDonald Institute for Archaeological Research, Cambridge, United Kingdom.

2012 Seeing Lithics. Oxbow Books, Oxford, United Kingdom.

Vaesen, Krist

2012 The Cognitive Bases of Human Tool Use. Behavioral and Brain Sciences 35(4):203-218.

Vallverdú, Josep, Manuel Vaquero, Isabel Cáceres, Ethel Allué, Jordi Rosell, Palmira Saladié, Gema Chacón, Andreu Ollé, Antoni Canals, Robert Sala, M. A. Courty, and Eudald Carbonell

2010 Sleeping Activity Area within the Site Structure of Archaic Human Groups: Evidence from Abric Romaní Level N Combustion Activity Areas. Current Anthropology 51:137-145.

Vernor, Vinge

1993 The Coming Technological Singularity. Whole Earth Review 81:88-95.

Vigilant, Linda, and Kevin E. Langergraber

2011 Inconclusive Evidence for Patrilocality in Neandertals. Proceedings of the National Academy of Sciences 108(18):E87.

Whiten, Andrew, Nicola McGuigan, Sarah Marshall-Pescini, and Lydia M. Hopper

2009 Emulation, Imitation, Over-Imitation and the Scope of Culture for Child and Chimpanzee. Philosophical Transactions of the Royal Society of London. Series B, Biological Sciences 364(1528):2417-2428.

Wimsatt, William

1986 Developmental Constraints, Generative Entrenchment, and the Innate-Acquired Distinction. In Integrating Scienti?c Disciplines, edited by William Bechtel, pp. 185-208. Martinus Nijhoff, Dordrecht, The Netherlands.

2001 Generative Entrenchment and the Developmental Systems Approach to Evolutionary Process. In Cycles of Contingency, edited by Susan Oyama and Paul E. Gri?ths, pp. 219-238. MIT Press, Cambridge, Massachusetts.
Wimsatt, William C., and James R. Griesemer

2007 Reproducing Entrenchments to Scaffold Culture: The Central Role of Development in Cultural Evolution. In Integrating Evolution and Development: From Theory to Practice, edited by Roger Sansom and Robert N. Brandon, pp. 227-323. MIT Press, Cambridge, Massachusetts.

\section{Notes}

1. Strictly speaking $\alpha$ is used by Henrich (2004:201) as the average error rate (how hard a skill is to learn; see Figure 1 ), while another parameter $\beta$ determines the variability of learning outcomes. The fraction $\alpha \beta$ is then taken to indicate the "complexity" of the skill. Like Powell et al. (2009), we suppress the $\beta$ parameter (implicitly assuming it to be constant), and noting that the unit of $\alpha$ is arbitrary, we refer, for simplicity, to $\alpha$ as "complexity."

2. Learning outcomes scatter depends on varying levels of creativity in the population, while the magnitude of the extent to which they scatter is seen as intrinsic to the skills, and is modeled by Henrich (2004) by the parameter $\beta$ (which is here suppressed and assumed to be constant.)

3. The Treadmill Model, notably, does not directly depict the evolution of skill complexity: it depicts the evolution of skillfulness, which is, in turn, argued logically to lead to evolutionary effects on skill complexity. Andersson and Read (2016) argue that the distinction between skillfulness and complexity, i.e., between $\alpha$ and $z$, has not been clear in the literature, with substantial confusion as a result.

4. In fact, the selection pressure for high levels of skillfulness $z$ in role models (skill bias) is maximally strong in the Treadmill Model: it is built into the ontology of the model by the choice of a Gumbel distribution (see Vaesen 2012 for an analysis of this choice).

5. What the Quasispecies Model has done in biology is indeed precisely to establish that the reason why this is possible is that transmission fidelity has become sufficiently high.

6. Fidelity in our interpretation encompasses the net effect of errors and the processes that serve to correct errors. This includes what Henrich (2004) means by "creativity" in the role of counteracting errors.

7. Or responsible for producing more complex culture once larger residential units were in place (regardless of what actually drove residential population size).

8. Parallels to the concept of a "technological singularity" are, however, tempting; see Vernor (1993)

Submitted January 27, 2015; Revised June 12, 2015; Revised October 11, 2015; Accepted October 13, 2015. 